\title{
Mental illness in patients with end-stage kidney disease in South Korea: a nationwide cohort study
}

\author{
Min-Jeong Lee ${ }^{1}$, Eunyoung Lee ${ }^{2,3,4}$, Bumhee Park ${ }^{2,3}$, Inwhee Park ${ }^{1}$ \\ ${ }^{1}$ Department of Nephrology, Ajou University School of Medicine, Suwon, Republic of Korea \\ ${ }^{2}$ Department of Biomedical Informatics, Ajou University School of Medicine, Suwon, Republic of Korea \\ ${ }^{3}$ Office of Biostatistics, Medical Research Collaborating Center, Ajou Research Institute for Innovative Medicine, Ajou University Medical Center, \\ Republic of Korea \\ ${ }^{4}$ Department of Medical Sciences, Biomedical Informatics, Graduate School of Ajou University, Suwon, Republic of Korea
}

Background: The limited literature on mental illness in end-stage kidney disease (ESKD) patients suggests that this disease is common and burdensome but underrecognized in clinical practice. This study aimed to analyze the prevalence of mental illness in ESKD patients.

Methods: We assessed the prevalence and patterns of mental illnesses in a nationwide cohort of patients diagnosed with ESKD between January 1, 2008, and December 31, 2017. The risk of mental illness was evaluated using a multivariable Cox proportional hazards model.

Results: A total of 70,079 patients met all study inclusion criteria. A total of $28.3 \%$ of patients had mental illness, and the specific distribution was as follows: depression, 16.8\%; anxiety, 20.0\%; somatoform/conversion disorder, 0.9\%; stress reaction/adjustment disorder, 2.5\%; and substance abuse disorder, $0.6 \%$. The frequency of mental illness was highest in patients on hemodialysis (HD), followed by patients on peritoneal dialysis (PD) and kidney transplant (KT) patients. The peak rate of mental illness in HD and PD patients was reached 1 to 2 years after renal replacement therapy initiation, but the peak rate of most mental illnesses in KT patients occurred before surgery. The prevalence of depression was 2.19 times higher in HD patients and 1.97 times higher in PD patients than in KT patients.

Conclusion: ESKD patients are at high risk of mental illness, and the prevalence of mental illness is highest in HD patients. Since the onset of mental illness occurs around the initiation of renal replacement therapy, clinicians need to pay attention to mental illness when treating ESKD patients.

Keywords: Anxiety, Chronic kidney failure, Depression, Mental disorders

Received: February 25, 2021; Revised: July 13, 2021; Accepted: August 23, 2021

Correspondence: Inwhee Park

Department of Nephrology, Ajou University School of Medicine, Worldcup-ro 164, Yeongtong-gu, Suwon 16499, Republic of Korea.

E-mail: inwhee@aumc.ac.kr

ORCID: https://orcid.org/0000-0002-9912-5393

Bumhee Park

Department of Biomedical Informatics, Ajou University School of Medicine, Worldcup-ro 164, Yeongtong-gu, Suwon 16499, Republic of Korea.

E-mail: bhpark@ajou.ac.kr

ORCID: https://orcid.org/0000-0002-5271-1571

Min-Jeong Lee and Eunyoung Lee contributed equally to this work as co-first authors.

Copyright (C) 2022 by The Korean Society of Nephrology

(a) This is an Open Access article distributed under the terms of the Creative Commons Attribution Non-Commercial and No Derivatives License (http:// creativecommons.org/licenses/by-nc-nd/4.0/) which permits unrestricted non-commercial use, distribution of the material without any modifications, and reproduction in any medium, provided the original works properly cited. 


\section{Introduction}

Patients with end-stage kidney disease (ESKD) experience a higher rate of mental illness than the general adult population $[1,2]$. Beyond depression, patients might experience a myriad of psychological distress symptoms including anxiety and fear of chronic kidney disease (CKD) progression (concerns about hopelessness, death, and dying). They also might experience recurrent psychological and physical trauma during the CKD course [3].

Previous large-scale studies have investigated the incidence and severity of psychiatric symptoms in ESKD patients $[4,5]$. A comprehensive analysis of psychiatric illnesses in a large, adult ESKD population found that $8.9 \%$ of patients on dialysis were hospitalized with a primary or secondary psychiatric diagnosis [4]. A systematic review and meta-analysis found a $1.4 \%-94.9 \%$ prevalence of depression in patients on dialysis, with a summary prevalence estimate of $39.3 \%$ when depression was assessed by questionnaire and $22.8 \%$ when assessed by interview [5]. Moreover, psychiatric information on ESKD patients remains underrecognized in clinical practice [6-8].

There are few studies on the prevalence of mental illness related to ESKD in Koreans. Since research on the relationship between ESKD and mental illness remains insufficient, we aimed to analyze the characteristics and prevalence of mental illness and psychiatric disorders in ESKD patients using data from the Health Insurance Review and Assessment (HIRA) service, a national registry including all patients in Korea.

\section{Methods}

The Republic of Korea public medical insurance system, the National Health Insurance Services (NHIS), includes the HIRA database of all health care claims for outpatient or inpatient visits to medical institutions and includes patient demographics, diagnoses, procedures, and prescriptions [9]. We retrospectively analyzed a nationwide cohort of patients diagnosed with ESKD between January 1, 2007, and December 31, 2017, from the HIRA database. This study was performed in accordance with the Declaration of Helsinki and the Institutional Review Board of Ajou University Hospital approved this study (No. AJIRB-MEDEXP-18-499), and the requirement for informed consent was waived as the NHIS database is anonymized according to strict confidentiality guidelines. Moreover, the attending government organization approved access to the HIRA database (HIRA No. M20181212478).

\section{Assessment of end-stage kidney disease population}

We collected the data of patients with at least one diagnosis code of CKD (International Classification of Diseases, 10th Revision [ICD-10], N18.0-18.6, 18.9) from 2007 to 2017, in Korea. We then selected ESKD patients who were newly diagnosed since 2008, so that we could observe them for at least 1 year before initiation of dialysis or transplant. The date of initial dialysis or transplant was defined as the index date. We hypothesized that anticipation regarding kidney replacement therapy (KRT) could affect the mental states of patients even before they actually started treatment. In addition, we wanted to observe changes in patient mental health when facing KRT initiation rather than the prevalence of newly developed mental illness after KRT initiation. Therefore, the observation period was defined as 1 year before the index date up to December 31, 2017. ESKD patients were confirmed by special exemption codes (V001: hemodialysis, HD; V003: peritoneal dialysis, PD; and V005: kidney transplant, KT) provided by the Korean government. Based on the special exemption codes and procedure codes (HD: O7020, O7021, O9991; PD: O707; and KT: R3280) on health claims, we classified patients into three KRT modality groups; HD, PD, and KT. Patients with less than 3 months of dialysis history and those who changed dialysis modality were excluded. For KT patients, preemptive KT cases were included in the KT group. Changes included: 1) change from PD to HD; 2) change to PD after being on HD for more than 3 months; 3 ) change from KT to HD or PD due to graft failure; or 4) transplantation surgery 1 year after the first dialysis initiation (for KT patients). Patients with no procedure codes related to KRT modalities or those younger than 18 years at the index date were excluded [10].

Baseline comorbidities, defined using ICD-10 codes, were observed in the year prior to the index date. The diagnostic codes of comorbidities were as follows: diabetes mellitus, E10-E14; hypertension, I10-I15; cardiovascular disease, I20-I25; cerebrovascular disease, I60-I69; chronic lung disease, J40-J47; and chronic liver disease, K70.3, 
K70.4, K72.1, K73, and K74.

\section{Assessment of mental illnesses}

We assessed five mental illnesses separately: i) depression, ii) anxiety, iii) somatoform/conversion disorder, iv) stress reaction/adjustment disorder, and v) substance abuse disorder. Each mental illness was identified by ICD-10 codes: F32 and F33 for depression, F40 and F41 for anxiety, F44 and F45 for somatoform/conversion disorder, F43 for stress reaction/ adjustment, and F10-F19 for substance abuse disorder [11-13]. Some patients experience co-occurring mental illnesses, so we created independent subcohorts for each mental illness to include all cases in the analysis. We examined all visits with a diagnosis code during the observation period and analyzed trends of mental illness prevalence over time.

\section{Statistical analysis}

We performed the analysis of variance for continuous variables and the chi-square test of homogeneity for categorical variables to compare baseline characteristics and the overall prevalence of mental illness between groups. For each KRT modality, the prevalence of each mental illness was presented as a percentage. We conducted McNemar test to examine any psychiatric prevalence changes before and after the index date by KRT modality. Density plots were used to observe the distribution of psychiatric visits over time, and the peaks indicated the highest patient prevalence. We assessed the risk of each mental illness using the Cox proportional hazards model with adjustments for age group and sex. Hazard ratios (HRs) and 95\% confidence intervals (CIs) were estimated for each covariate. All statistical analyses were two-sided and performed using SAS version 9.4 (SAS Institute, Cary, NC, USA). A p-value of $<0.05$ was considered statistically significant.

\section{Results}

\section{Baseline characteristics of ESKD cohort}

The study flowchart is presented in Fig. 1. The data of 813,907 patients with a diagnosis code of ESKD were extracted from the HIRA database. Of those, 108,264 ESKD patients registered between 2008 and 2017, were selected. After excluding 38,185 patients based on the exclusion criteria, the final ESKD cohort comprised 70,079 patients, including 61,497 (87.8\%) in the HD group, 3,901 (5.6\%) in the KT group, and 4,681 (6.7\%) in the PD group. Fig. 1 shows the mental illness distribution according to KRT modality. The baseline characteristics of the study population are described in Table 1. Patients were followed for $4.3 \pm 2.4$ years. Overall, the mean age at the index date was $60.6 \pm$ 14.2 years. The HD group was the oldest (mean age, $62.1 \pm$ 13.7 years), and most KT and PD patients were aged 40 to 59 years. ESKD was more prevalent in males $(59.6 \%)$ than in females. During the year before the index date, anxiety was the most prevalent disorder, with the KT group showing the highest prevalence. Comorbidities observed in the year before the index date were common, especially in the HD group, and the most prevalent comorbidities were hypertension (94.7\%) and diabetes mellitus (74.4\%).

\section{Mental illness by kidney replacement therapy modality}

Among all ESKD patients, 19,823 (28.3\%) had at least one psychiatric diagnosis code during the observation period. The most frequent mental illness was anxiety $(14,033$, $20.0 \%)$, followed by depression $(11,797,16.8 \%)$, stress reaction/ adjustment disorder $(1,776,2.5 \%)$, somatoform/conversion disorder $(626,0.9 \%)$, and substance abuse disorder $(399,0.6 \%)$. This order of frequency remained the same for all KRT modalities. Anxiety was still the most prevalent mental illness (HD, 12,721 [20.7\%]; KT, 524 [13.4\%]; and PD, 788 [16.8\%]), followed by depression (HD, 10,721 [17.4\%]; KT, 312 [8.0\%]; and PD, 764 [16.3\%]). The frequencies of all mental illnesses, except for somatoform/conversion disorder, were highest in the HD group, followed by the PD and KT groups. The prevalence of somatoform/conversion disorder was higher in the KT group than in other groups (Fig. 2).

\section{Changes in mental illness before and after end-stage kid- ney disease diagnosis}

We assessed whether a significant change in the prevalence of mental illness had occurred before and after the index date (Supplementary Table 1, available online). Overall, ESKD patients had more mental illnesses after the index date than before, with 4.9 times more depression, 4.0 times 


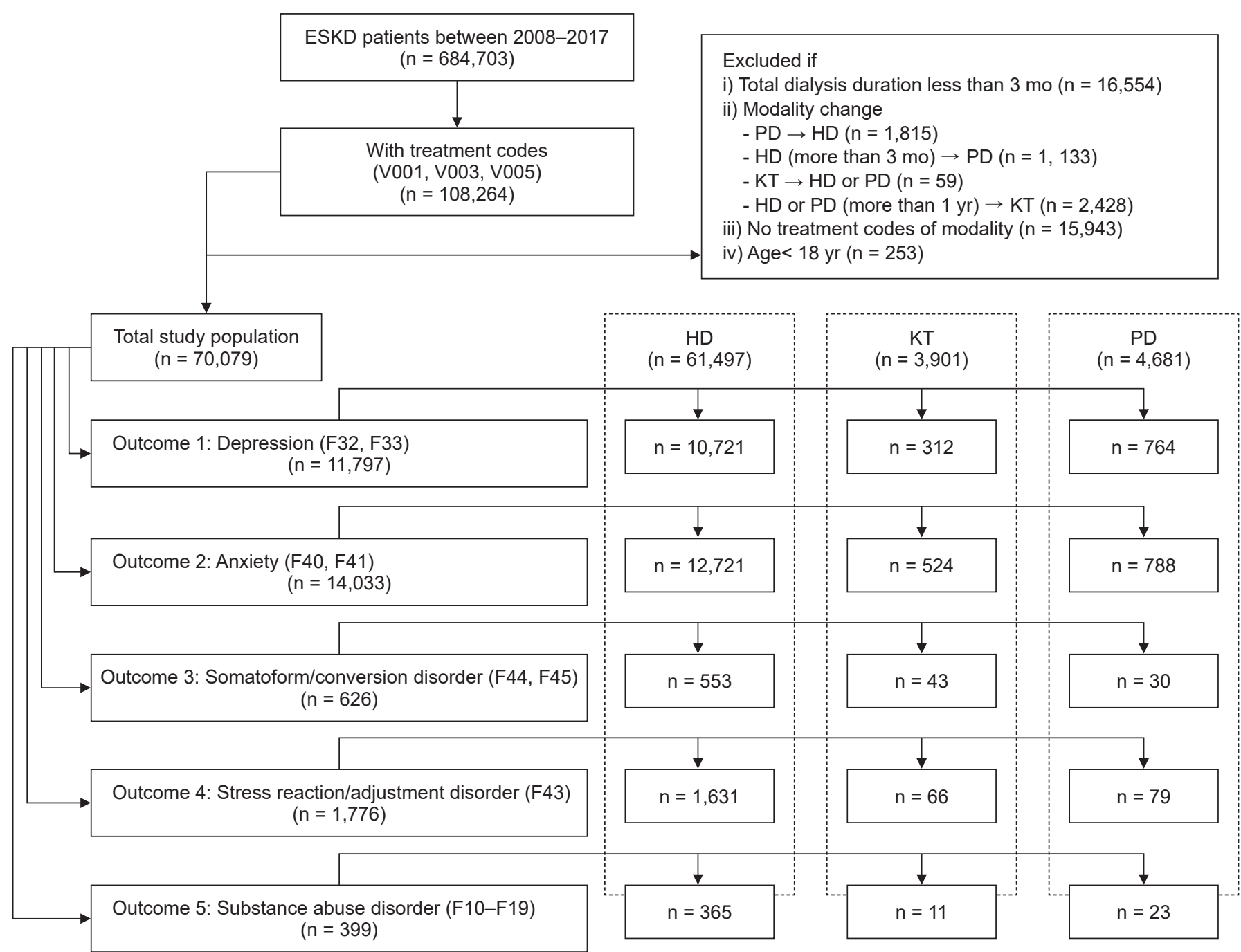

Figure 1. Flowchart of the study cohort.

ESKD, end-stage kidney disease; HD, hemodialysis; KT, kidney transplant; PD, peritoneal dialysis.

more anxiety, 3.5 times more somatoform/conversion disorder, 2.9 times more stress reaction/adjustment disorder, and 2.0 times more substance abuse disorder (all $\mathrm{p}<0.001$ ). However, the prevalence of somatoform/conversion disorders in the KT group and substance abuse disorders in the KT and PD groups did not show significant change after the index date. The KT group showed the smallest change after the index date, while the HD group showed the largest change; $3 \%$ of the patients in the HD group had depression before the index date, but $16.0 \%$ had depression after the index date $(\mathrm{p}<0.001)$.

\section{Frequency density of mental illnesses}

The frequency density plot of mental illness among ESKD patients is shown in Fig. 3. The overall frequency of mental illness started to increase 1 year before the index date. There was a distinct difference in the distribution of mental illnesses (Fig. 3). The KT group, except for substance abuse disorder patients, had the highest number of psychiatric visits prior to KT. In the HD group, substance abuse disorders peaked around 5 months and other mental illnesses around 2 years after dialysis initiation. In the PD group, most of the peak times for mental illnesses, excluding stress 
Table 1. Baseline characteristics of the end-stage kidney disease cohort

\begin{tabular}{|c|c|c|c|c|c|}
\hline Characteristic & Overall $(n=70,079)$ & $\mathrm{HD}(\mathrm{n}=61,497)$ & $\mathrm{KT}(\mathrm{n}=3,901)$ & $P D(n=4,681)$ & p-value \\
\hline Follow-up (yr) & $4.3 \pm 2.4$ & $4.2 \pm 2.4$ & $4.4 \pm 2.3$ & $4.5 \pm 2.3$ & $<0.001$ \\
\hline Age at index date (yr) & $60.6 \pm 14.2$ & $62.1 \pm 13.7$ & $45.0 \pm 11.7$ & $53.9 \pm 13.4$ & $<0.001$ \\
\hline $18-39$ & $5,840(8.3)$ & $3,876(6.3)$ & 1,288 (33.0) & $676(14.4)$ & $<0.001$ \\
\hline $40-59$ & $25,729(36.7)$ & 21,115 (34.3) & $2,229(57.1)$ & $2,385(51.0)$ & \\
\hline$\geq 60$ & $38,510(55.0)$ & $36,506(59.4)$ & $384(9.9)$ & $1,620(34.6)$ & \\
\hline Male sex & $41,777(59.6)$ & $36,842(59.9)$ & $2,291(58.7)$ & $2,644(56.5)$ & $<0.001$ \\
\hline \multicolumn{6}{|l|}{ Comorbidity $^{\mathrm{b}}$} \\
\hline Diabetes & $46,649(66.6)$ & $41,934(68.2)$ & $1,814(46.5)$ & $2,901(62.0)$ & $<0.001$ \\
\hline Hypertension & $64,299(91.8)$ & $56,258(91.5)$ & $3,722(95.4)$ & 4,319 (92.3) & $<0.001$ \\
\hline Cardiovascular & $18,085(25.8)$ & $16,257(26.4)$ & $639(16.4)$ & $1,189(25.4)$ & $<0.001$ \\
\hline Cerebrovascular & $11,671(16.7)$ & $10,961(17.8)$ & $220(5.6)$ & $490(10.5)$ & $<0.001$ \\
\hline Chronic lung disease & $15,937(22.7)$ & $14,594(23.7)$ & $594(15.2)$ & 749 (16.0) & $<0.001$ \\
\hline Chronic liver disease & $3,219(4.6)$ & $2,956(4.8)$ & $81(2.1)$ & $182(3.9)$ & $<0.001$ \\
\hline \multicolumn{6}{|l|}{ Psychiatric disorder ${ }^{b}$} \\
\hline Depression & 2,181 (3.1) & $1,860(3.0)$ & $151(3.9)$ & $170(3.6)$ & 0.001 \\
\hline Anxiety & $3,064(4.4)$ & $2,584(4.2)$ & $258(6.6)$ & $222(4.7)$ & $<0.001$ \\
\hline Somatoform/conversion disorder & $125(0.2)$ & $98(0.2)$ & $21(0.5)$ & $6(0.1)$ & $<0.001$ \\
\hline Stress reaction/adjustment disorder & $477(0.7)$ & $424(0.7)$ & $24(0.6)$ & $29(0.6)$ & 0.75 \\
\hline Substance abuse disorder & $133(0.2)$ & $119(0.2)$ & $6(0.2)$ & $8(0.2)$ & 0.82 \\
\hline
\end{tabular}

Data are expressed as mean \pm standard deviation or number (\%).

$\mathrm{HD}$, hemodialysis; KT, kidney transplant; PD, peritoneal dialysis.

${ }^{a} \mathrm{p}$-value was based on the analysis of variance test; otherwise, the chi-square test was used. ${ }^{\mathrm{b}}$ Comorbidities and psychiatric disorders observed during the observation period, which was 1 year before initiation of renal replacement therapy to December 31, 2017.

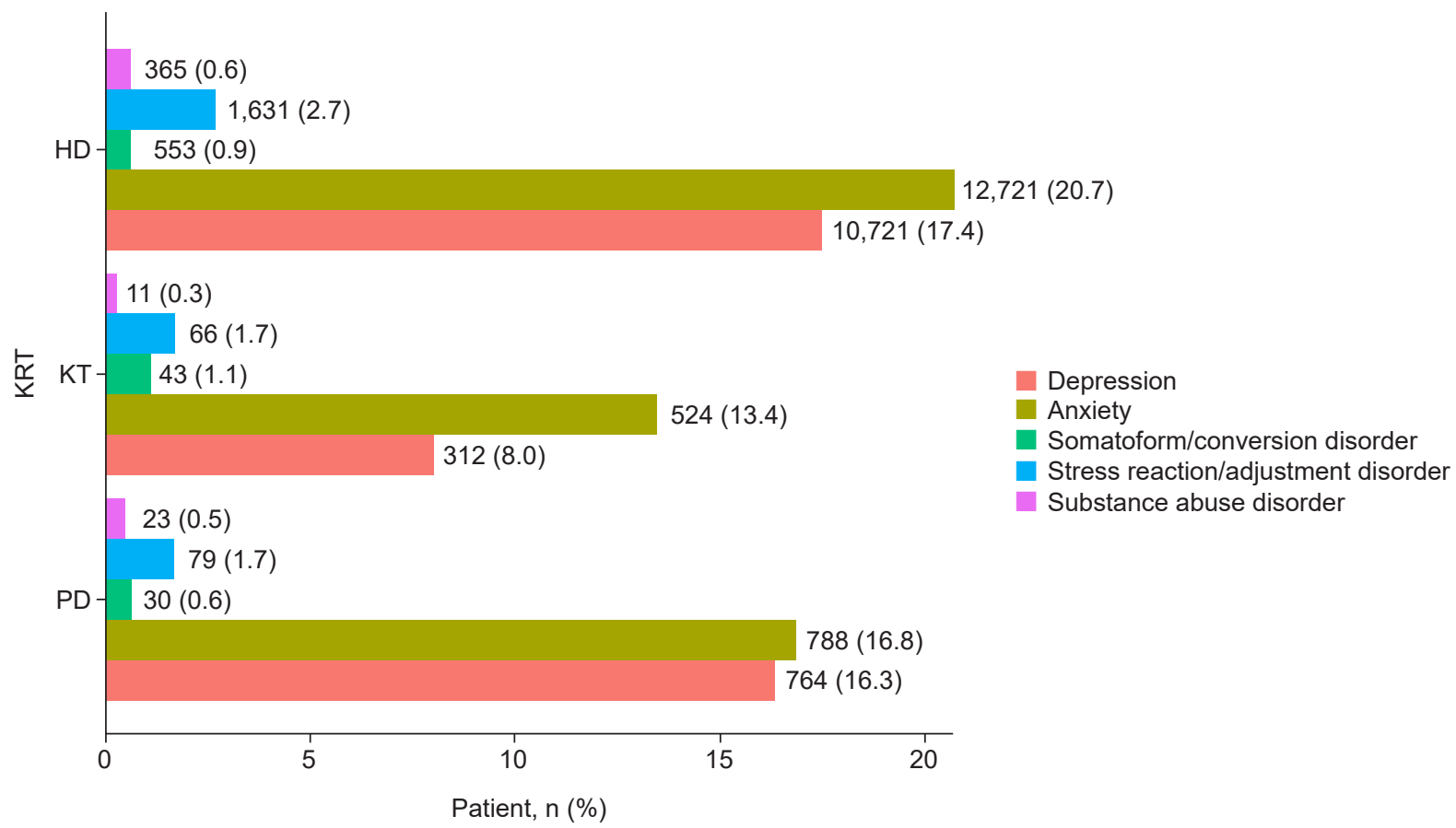

Figure 2. Mental illnesses in end-stage kidney disease patients by kidney replacement therapy.

KRT, kidney replacement therapy; HD, hemodialysis; KT, kidney transplant; PD, peritoneal dialysis. 

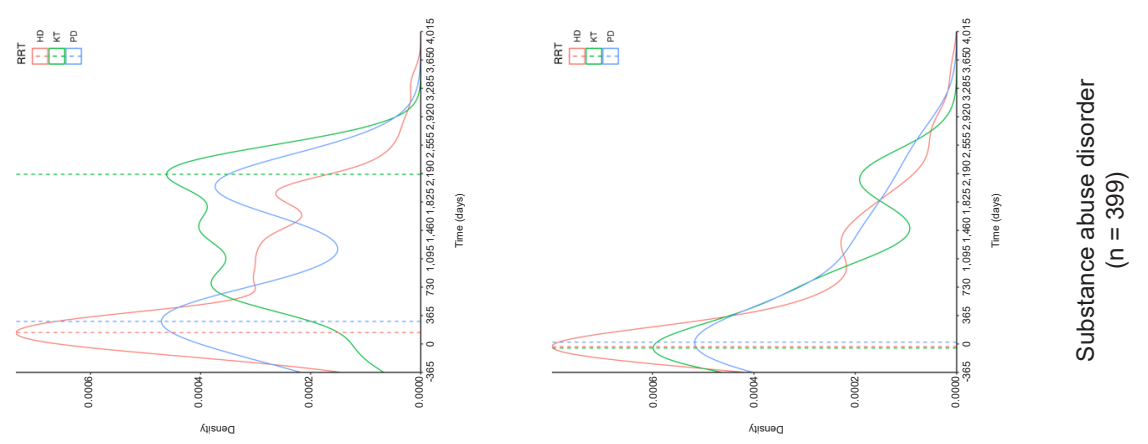

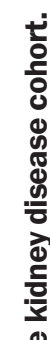
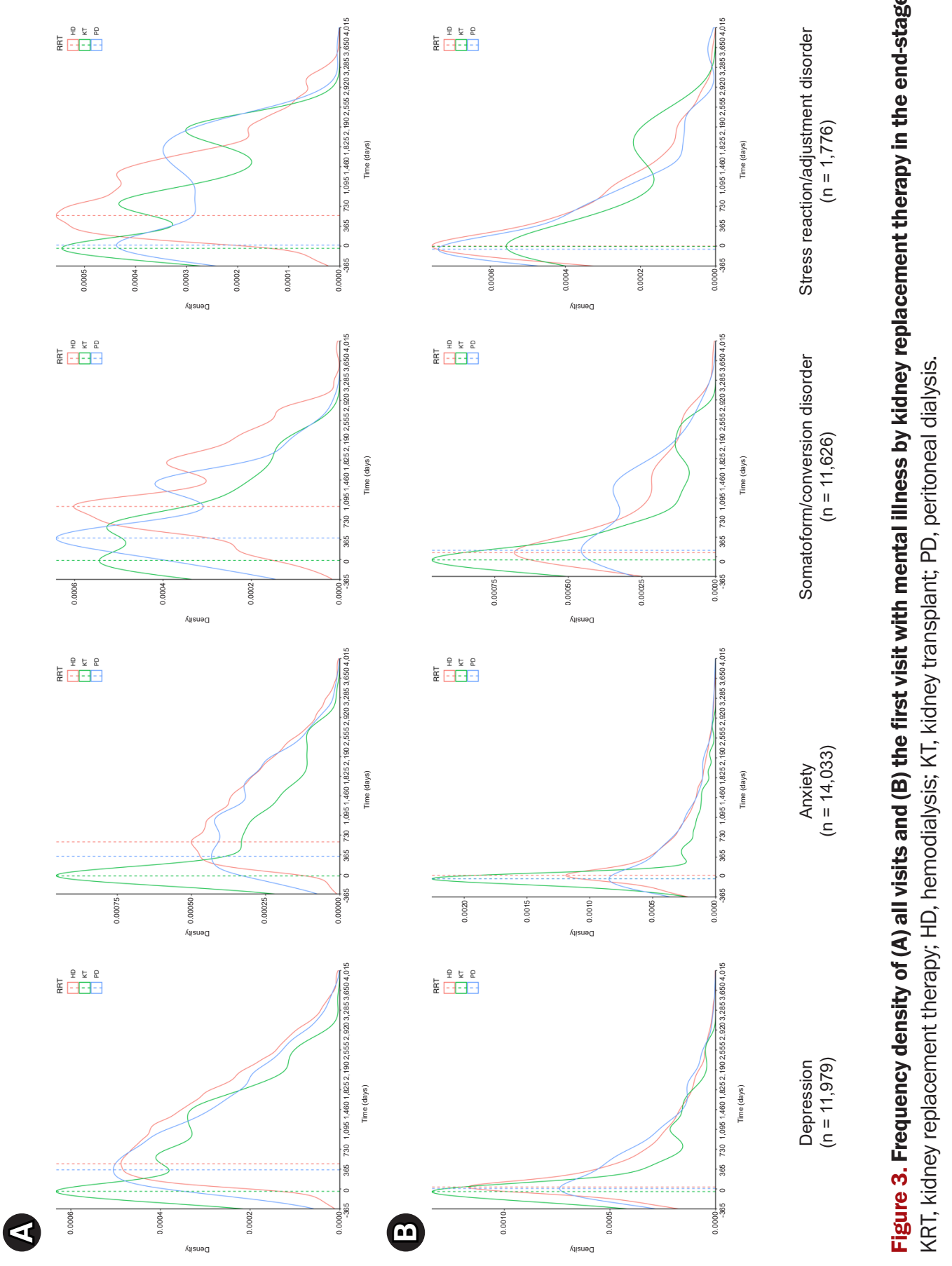
reaction/adjustment disorder, were reached around 1 year after KRT initiation.

Frequency density plots were constructed from the frequency of the first onset of mental illnesses (Fig. 3B). The most common time to receive the first treatment for most mental illnesses was within 1 to 2 months before or after initiation of KRT, the transitional period. A higher frequency of depression, anxiety, and stress reaction/adjustment disorder around KRT initiation was noted. The computed peak time of each density plot is provided in Supplementary Table 2 (available online).

\section{Hazard ratio of mental illnesses}

Hazard ratio of mental illnesses was shown in Table 2. The age- and sex-adjusted risk of depression was higher in the HD (adjusted HR [aHR], 2.19; 95\% CI, 1.95-2.46; p < 0.001) and PD (aHR, 1.97; 95\% CI, 1.73-2.26; $\mathrm{p}<0.001$ ) groups than in the KT group. With age, the risk of depression increased significantly, and patients older than 60 years had a 1.27-times higher risk than those younger than 40 years $(\mathrm{p}<0.001)$. Depression was significantly more common in males than in females (aHR, 1.17; 95\% CI, 1.128-1.216; $\mathrm{p}<$ 0.001 ). For anxiety, the HD (aHR, 1.64; 95\% CI, 1.50-1.80; $\mathrm{p}<0.001$ ) and PD (aHR, 1.22; 95\% CI, 1.09-1.35; p < 0.001) groups had a significantly higher risk than the KT group. Patients aged 40 to 59 years had a 1.11-times higher risk of anxiety than did younger patients $(\mathrm{p}=0.002)$. Similar to depression, males had a higher risk of anxiety than females (aHR, 1.20; 95\% CI, 1.16-1.24; $\mathrm{p}<0.001$ ). The HD group was at the highest risk for stress reaction/ adjustment and substance abuse disorders. The KT group was at the lowest risk for most mental illnesses. However, the somatoform/ conversion disorder risk was exceptionally high in the KT group, 1.7 times higher than that in the PD group $(\mathrm{p}=0.04)$.

\section{Discussion}

In our study, the prevalence of mental illnesses was $28.3 \%$ in ESKD patients, with anxiety $(20.0 \%)$ and depression (16.8\%) being the most common. The prevalence of mental illness was highest in HD patients, followed by PD and KT patients. Since many ESKD patients seek mental illness-related treatment before and after initiation of KRT, it is necessary to pay attention to mental illnesses at the initiation of KRT. Diagnosis and treatment for ESKD can induce emotional stress, which can affect the prognosis of the disease $[14,15]$. Mental illness can be both a consequence of life on dialysis and a root cause of CKD and ESKD. The lack of self-care and energy that characterize depression has detrimental effects on patients' ability to cope with the disease.

The prevalence of depression in a sampled population of one million individuals in South Korea, was 5.3\% in 2013 [16], and the prevalence of depression was $6.7 \%$ from the 2014 Korea National Health and Nutrition Examination Survey [17]. However, previous studies of mental illness in ESKD patients showed a higher prevalence of mental illnesses. Chilcot et al. [18] reported that $20 \%$ to $30 \%$ of ESKD patients have significant depressive symptoms, which is higher than the lifetime prevalence of depression of approximately $6.9 \%$ observed in the general population and the mean prevalence of approximately $17 \%$ seen in cancer patients [19-21]. The prevalence of psychiatric morbidity in our ESKD cohort was $28.3 \%$. Many previous studies have reported a higher prevalence of mental illness than ours. One Brazilian study reported a psychiatric morbidity rate of $46.4 \%$ in patients undergoing dialysis and demonstrated that psychiatric disorders can negatively impact the quality of life and treatment compliance [22]. Kim [23] reported in 2010 , that the prevalence of depression, anxiety, and concomitant depression and anxiety was $58.5 \%, 27.9 \%$, and $26.0 \%$, respectively. In the HD group in our study, the anxiety and depression rates were $20.7 \%$ and $17.4 \%$, respectively. In the previous studies [22,23], all patients underwent the Mini International Neuropsychiatric Interview or Hospital Anxiety and Depression Scale as a screening test at a single HD center to directly evaluate mental illness. In our study, we identified patients treated for mental illness using a claims database. Hence, mental illness is not difficult to recognize, and it is possible that treatment might have been insufficient compared to the actual prevalence.

The prevalence of mental illness differs according to KRT modality, and the timing at which it occurs also differs, particularly in the case of HD patients where the change in mental illness before and after dialysis was most obvious. Once ESKD is diagnosed, patients are subject to many stressors. They spend at least 3 days/week in a dialysis center or in a hospital for about 4 hours each session, they undergo dietary and lifestyle changes, and they need to manage their daily 
Table 2. Hazard ratios of mental illnesses from the multivariable Cox proportional hazards model in the end-stage kidney disease cohort $(n=70,079)$

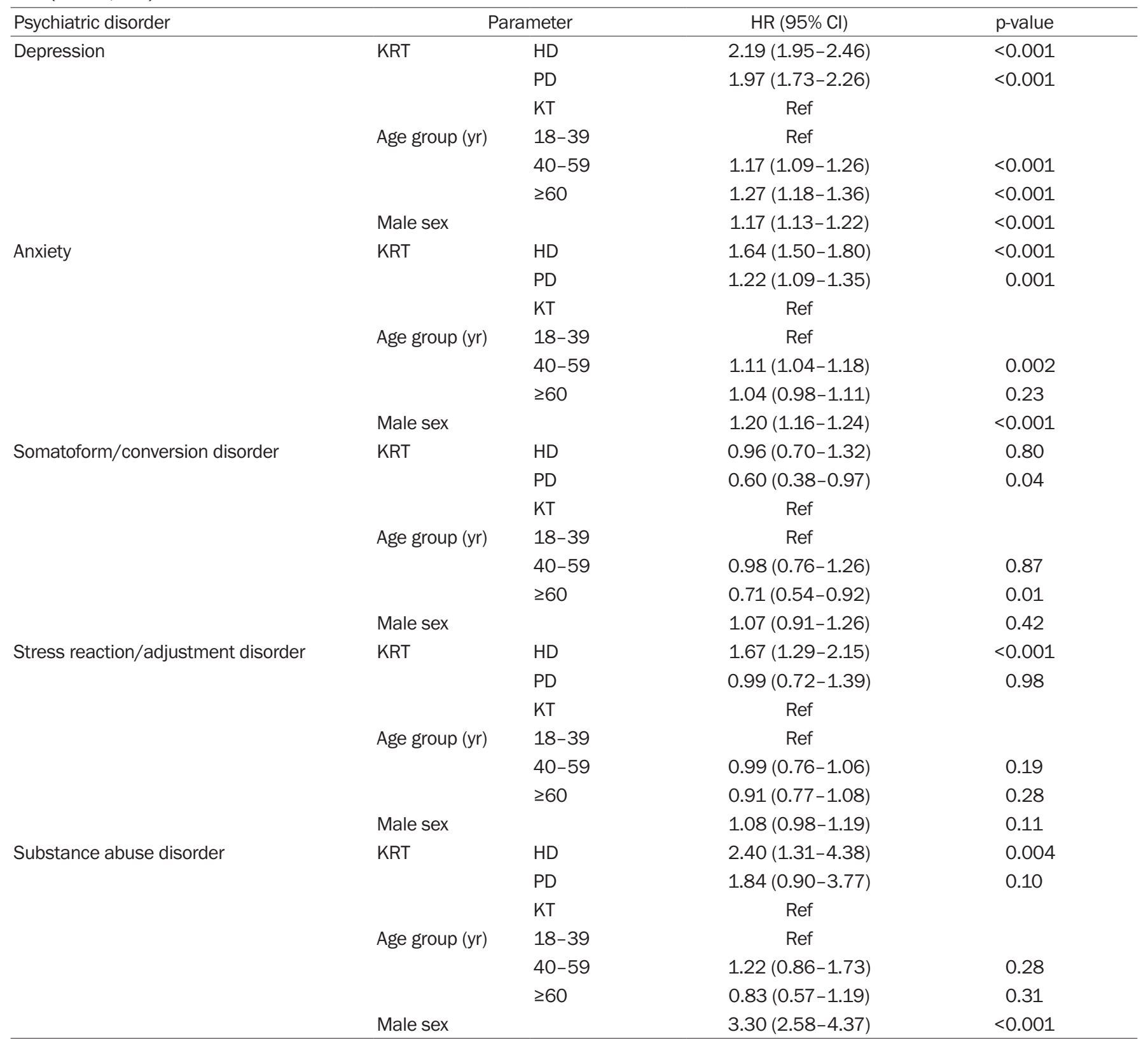

$\mathrm{HR}$, hazard ratio; Cl, confidence interval; HD, hemodialysis; KT, kidney transplant; KRT, kidney replacement therapy; PD, peritoneal dialysis; Ref, reference group.

trips to the dialysis unit, in addition to considering possible family relocations and financial restrictions. Since HD patients visit the hospital three times a week and have higher contact with dialysis unit staff compared to patients on other KRT modalities, more active monitoring of HD patients by dialysis unit staff is required [24].

In this study, the prevalence of depression and anxiety was high within 1 to 2 years of dialysis, and the peak time of the first treatment was within 1 to 2 months of dialysis. Some patients undergo planned dialysis at KRT initiation, but others require urgent dialysis. In urgent cases, the stress of not only the patient, but also the medical staff is significant, and mental health aspects are likely to be overlooked. Therefore, nephrologists also need to pay more attention to the mental 
illness aspects around the timing of KRT initiation.

KT is considered the most effective treatment for ESKD patients. In addition to somatic benefits, such as reduced cardiovascular risk and overall mortality, patients also appear to benefit regarding individual health-related quality of life. However, transplantation is a very demanding and particularly stressful event that requires the patient to implement his biopsychosocial skills to accept and integrate the new organ physically and mentally. Therefore, KT involves numerous psychological, existential, affective, relational, and social changes for the patients and their families $[25,26]$. During the pre-evaluation prior to KT surgery, there is intervention with social work teams and psychiatrists. Therefore, it might be easier to assess mental illness in KT patients than in HD or PD patients. Among KT patients in our study, the depression and anxiety rates were $8.5 \%$ and $13.4 \%$, respectively, representing the most common mental illnesses in this group. Other studies have reported anxiety and depression as the most likely disorders in KT recipients to influence disease process and graft survival [27]. To improve the quality of life of recipients, the management of mental illness is important because sufficient attention to treatment compliance, including drug compliance, is required.

One interesting result was that the prevalence of mental illnesses such as depression and anxiety, which usually is higher in women than in men in the general population [28-30], was higher in male ESKD patients in this study. Males had a 1.17 times higher risk of depression, 1.20 times higher risk of anxiety, and 3.3 times higher risk of substance abuse disorder than females. In another study of ESKD patients, there was no significant sex difference in the prevalence of mental illness [31]. In a United States ESKD study, the rates of depression and anxiety were higher in women than in men, although alcohol-related mental disorders were more prevalent in men than in women [32]. Compared to other studies, ours showed that Korean ESKD men have a higher prevalence of depression and anxiety than Korean women. This is probably because, in Korea, there is a more male-dependent home economic structure than in Western countries, so depression and anxiety occur due to physical and social constraints [33,34].

Although our study did not analyze mortality of ESKD patients, previous studies have reported that mental illness is associated with quality of life and/or mortality [35-39].
Patient nonadherence and psychological distress are highly prevalent among ESRD patients, and both contribute to greater morbidity and earlier mortality in this population. The number of patients with ESKD is increasing gradually $[40,41]$. Therefore, further studies should be conducted to evaluate more clearly the effectiveness of psychological interventions and to clarify the role of depression and social support on patient mortality.

There are a few limitations to this study. First, we examined mental illness cases using HIRA claims data, which offered only codes and demographic information. Clinical data (laboratory data), social data (educational data, marital status), and psychosocial factors were not available. Second, the disease code was based on claims data based on the treatment environment and was not created for a research setting. Moreover, the diagnostic accuracy of mental illness using ICD codes is not as high as that of structured clinical interviews using questionnaires. Furthermore, the diagnostic codes for mental illness can be influenced by medications, such as antidepressants prescribed for insomnia. Also, nephrologists tend to use psychiatric drugs frequently for controlling itching and pain in ESKD patients. However, in Korea, there is a tendency to refuse treatment for fear of being labeled mentally ill [42]. Therefore, diagnoses of mental illnesses are made very conservatively, which can compensate for the potential biases that would have arisen if patients had comorbid psychiatric disorders or symptoms. Third, the analysis was conducted under the assumption that the date of input of the diagnosis code was the actual diagnosis date of mental illness. Time discrepancies occur between diagnoses entered in the data and diseases that a patient has in reality, and this can be a source of limitation of HIRA data. Fourth, since the observation period was defined as 1 year before KRT initiation to December 31,2017 , there is a limitation in not considering the period of mental illness.

The strengths of our study are that it was a 10-year nationwide study of the entire population and an assessment of the prevalence of mental illness in ESKD patients according to KRT modality. To supplement the limited data of our study, further studies are needed to clarify mental illnesses based on the Diagnostic and Statistical Manual of Mental Disorders (DSM), prescribed psychiatric medications, and mortality. Furthermore, proper approaches for therapeutic interventions must be identified. Randomized, 
controlled treatment trials in patients with ESKD are needed, as mental health is a modifiable risk factor for poor outcomes that nephrologists and mental health care workers can address.

In conclusion, ESKD patients have a high prevalence of mental illness, and HD patients have a higher prevalence of mental illness than do PD and KT patients. Furthermore, since there are many cases of mental illness-related treatment around KRT initiation, we must pay attention to mental illness as well as physical problems when treating ESKD patients.

\section{Conflicts of interest}

All authors have no conflicts of interest to declare.

\section{Funding}

This study was supported by research grants from Daewon Pharmaceutical Company and the National Research Foundation of Korea (2019R1G1A1100671). The study sponsors had no role in study design, analysis, or interpretation of data.

\section{Authors' contributions}

Conceptualization: PI, PB

Data curation: MJL

Formal analysis: EL

Funding acquisition: PI

Investigation: EL

Writing-original draft: MJL, EL

Writing-review \& editing: PI, PB, MJL

All authors read and approved the final manuscript.

\section{ORCID}

Min-Jeong Lee, https://orcid.org/0000-0002-2611-7333

Eunyoung Lee, https://orcid.org/0000-0002-9440-9119

Bumhee Park, https://orcid.org/0000-0002-5271-1571

Inwhee Park, https://orcid.org/0000-0002-9912-5393

\section{References}

1. Lopes AA, Albert JM, Young EW, et al. Screening for depression in hemodialysis patients: associations with diagnosis, treatment, and outcomes in the DOPPS. Kidney Int 2004;66:2047-2053.

2. DeOreo PB. Hemodialysis patient-assessed functional health status predicts continued survival, hospitalization, and dialysis-attendance compliance. Am J Kidney Dis 1997;30:204-212.

3. Novak M, Mucsi I, Mendelssohn DC. Screening for depression: only one piece of the puzzle. Nephrol Dial Transplant 2013;28:1336-1340.

4. Kimmel PL, Thamer M, Richard CM, Ray NF. Psychiatric illness in patients with end-stage renal disease. Am J Med 1998;105:214-221.

5. Palmer S, Vecchio M, Craig JC, et al. Prevalence of depression in chronic kidney disease: systematic review and meta-analysis of observational studies. Kidney Int 2013;84:179-191.

6. Cukor D, Cohen SD, Peterson RA, Kimmel PL. Psychosocial aspects of chronic disease: ESRD as a paradigmatic illness. J Am Soc Nephrol 2007;18:3042-3055.

7. Cohen SD, Cukor D, Kimmel PL. Anxiety in patients treated with hemodialysis. Clin J Am Soc Nephrol 2016;11:2250-2255.

8. Watnick S, Wang PL, Demadura T, Ganzini L. Validation of 2 depression screening tools in dialysis patients. Am J Kidney Dis 2005;46:919-924.

9. Kim JA, Yoon S, Kim LY, Kim DS. Towards actualizing the value potential of Korea Health Insurance Review and Assessment (HIRA) data as a resource for health research: strengths, limitations, applications, and strategies for optimal use of HIRA data. $J$ Korean Med Sci 2017;32:718-728.

10. Lee MJ, Lee E, Park B, Park I. Epidemiological characteristics of cancers in patients with end-stage kidney disease: a Korean nationwide study. Sci Rep 2021;11:3929.

11. Heo J, Chun M, Oh YT, Noh OK. Psychiatric comorbidities among endometrial cancer survivors in South Korea: a nationwide population-based, longitudinal study. J Gynecol Oncol 2019;30:e15.

12. Heo J, Noh OK, Oh YT, Chun M, Kim L. Psychiatric comorbidities among patients undergoing liver transplantation in South Korea: a nationwide population-based study. Hepatol Int 2018;12:174-180.

13. Heo J, Chun M, Oh YT, Noh OK, Kim L. Psychiatric comorbidities among ovarian cancer survivors in South Korea: a nationwide population-based, longitudinal study. Psychooncology 2018;27:1021-1026.

14. Kimmel PL, Peterson RA, Weihs KL, et al. Psychosocial factors, behavioral compliance and survival in urban hemodialysis patients. Kidney Int 1998;54:245-254. 
15. Finkelstein FO, Finkelstein SH. Depression in chronic dialysis patients: assessment and treatment. Nephrol Dial Transplant 2000;15:1911-1913.

16. Kim GE, Jo MW, Shin YW. Increased prevalence of depression in South Korea from 2002 to 2013. Sci Rep 2020;10:16979.

17. Shin C, Kim Y, Park S, et al. Prevalence and associated factors of depression in general population of Korea: results from the Korea National Health and Nutrition Examination Survey, 2014. J Korean Med Sci 2017;32:1861-1869.

18. Chilcot J, Wellsted D, Farrington K. Depression in end-stage renal disease: current advances and research. Semin Dial 2010;23:74-82.

19. Bossola M, Ciciarelli C, Di Stasio E, et al. Symptoms of depression and anxiety over time in chronic hemodialysis patients. $J$ Nephrol 2012;25:689-698.

20. Bromet E, Andrade LH, Hwang I, et al. Cross-national epidemiology of DSM-IV major depressive episode. BMC Med 2011;9:90.

21. Krebber AM, Buffart LM, Kleijn G, et al. Prevalence of depression in cancer patients: a meta-analysis of diagnostic interviews and self-report instruments. Psychooncology 2014;23:121-130.

22. Martiny C, e Silva AC, Neto JP, Nardi AE. Psychiatric disorders in patients with end-stage renal disease. J Ren Care 2012;38:131137.

23. Kim SR. Depression and anxiety in maintenance hemodialysis patients: a single center study. Korean J Nephrol 2010;29:733-741.

24. Finnegan-John J, Thomas VJ. The psychosocial experience of patients with end-stage renal disease and its impact on quality of life: findings from a needs assessment to shape a service. ISRN Nephrol 2012;2013:308986.

25. De Pasquale C, Pistorio ML, Veroux M, et al. Psychological and psychopathological aspects of kidney transplantation: a systematic review. Front Psychiatry 2020;11:106.

26. Bello BT, Raji YR. Knowledge, attitudes and beliefs of first-degree relatives of patients with chronic kidney disease toward kidney donation in Nigeria. Saudi J Kidney Dis Transpl 2016;27:118-124.

27. Fukunishi I, Aikawa A, Ohara T, Hasegawa A. Psychiatric problems among recipients of kidney transplants: a 10-year follow-up. Transplant Proc 2002;34:2766.

28. Kuehner C. Why is depression more common among women than among men? Lancet Psychiatry 2017;4:146-158.

29. Kiely KM, Brady B, Byles J. Gender, mental health and ageing. Maturitas 2019;129:76-84.

30. Baxter AJ, Scott KM, Ferrari AJ, Norman RE, Vos T, Whiteford
HA. Challenging the myth of an "epidemic" of common mental disorders: trends in the global prevalence of anxiety and depression between 1990 and 2010. Depress Anxiety 2014;31:506-516.

31. Khan A, Khan AH, Adnan AS, Sulaiman SAS, Mushtaq S. Prevalence and predictors of depression among hemodialysis patients: a prospective follow-up study. BMC Public Health 2019;19:531.

32. Kimmel PL, Fwu CW, Abbott KC, et al. Psychiatric illness and mortality in hospitalized ESKD dialysis patients. Clin J Am Soc Nephrol 2019;14:1363-1371.

33. Kim S, Bryant FB. The influence of gender and cultural values on savoring in Korean undergraduates. Int J Wellbeing 2017;7:4363.

34. Kim SS, Kim-Godwin YS. Cultural context of family religiosity/ spirituality among Korean-American elderly families. J Cross Cult Gerontol 2019;34:51-65.

35. Covinsky KE, Kahana E, Chin MH, Palmer RM, Fortinsky RH, Landefeld CS. Depressive symptoms and 3-year mortality in older hospitalized medical patients. Ann Intern Med 1999;130:563569.

36. Ruberman W, Weinblatt E, Goldberg JD, Chaudhary BS. Psychosocial influences on mortality after myocardial infarction. N Engl J Med 1984;311:552-559.

37. Foster FG, Cohn GL, McKegney FP. Psychobiologic factors and individual survival on chronic renal hemodialysis-a two year follow-up: Part I. Psychosom Med 1973;35:64-82.

38. Christensen AJ, Ehlers SL. Psychological factors in end-stage renal disease: an emerging context for behavioral medicine research. J Consult Clin Psychol 2002;70:712-724.

39. Bujang MA, Musa R, Liu WJ, Chew TF, Lim CT, Morad Z. Depression, anxiety and stress among patients with dialysis and the association with quality of life. Asian J Psychiatr 2015;18:49-52.

40. GBD Chronic Kidney Disease Collaboration. Global, regional, and national burden of chronic kidney disease, 1990-2017: a systematic analysis for the Global Burden of Disease Study 2017. Lancet 2020;395:709-733.

41. Yang CW, Harris DC, Luyckx VA, et al. Global case studies for chronic kidney disease/end-stage kidney disease care. Kidney Int Suppl (2011) 2020;10:e24-e48.

42. Jo SJ, Yim HW, Jeong H, et al. Prevalence of depressive disorder of outpatients visiting two primary care settings. J Prev Med Public Health 2015;48:257-263. 